\title{
Electrochemical Detection of Arsenic Using Modified Platinum-Cobalt Electrode
}

\author{
Nurulhaidah Daud, Nur Khairul Nabila Kamaruddin, Suraya Sulaiman, and Mohd Ismahadi Syono
}

\begin{abstract}
An electrochemical detection of arsenic(III) solution was investigated by using a modified cobalt deposited on a silicon-based platinum electrode. The modified platinum-cobalt electrodes were prepared via electrode position process in varied deposition times. Surface morphology of modified electrode was investigated through FESEM. An electrocatalytic activity of arsenic solution on each deposited electrodes was examined with cyclic voltammetry $(\mathrm{CV})$ at a potential range of $-1.5 \mathrm{~V}$ to $+1.5 \mathrm{~V}$ (vs. pseudo $\mathrm{Ag} / \mathrm{AgCl}$ ) in $0.1 \mathrm{M}$ KOH supporting electrolyte. A voltammogram of arsenic(III) in different concentration was plotted and showed an increased peak current of arsenic(III) reduction, suggesting that the cobalt has high catalytic ability for arsenic reduction. $R^{2}$ values obtained from the linear regression equation of a modified Platinum-Cobalt electrode in 10s and 20s deposition time were 0.9976 and 0.9815 , respectively. Various electrodeposition times showed a different morphology on electrode surfaces and affected the current signal of arsenic(III) reduction peak in cyclic voltammetry.
\end{abstract}

Index Terms-Arsenic, cobalt electrode, cyclic voltammetry, electrodeposition, modified electrode.

\section{INTRODUCTION}

Arsenic (As) is a toxic metal that can only be transformed into a form that is less toxic when exposed to living organism in the environment. It can be categorized as organic or inorganic compounds that can vary in their solubility, mobility, and toxicity. The toxicity level of arsenic compound depends on their different valence states. In environment, the main forms are arsenic(III) (arsenite, As(III)) and arsenic(V) oxyacid, and can be deprotonated to arsenate anions. As the second most toxic metal after lead, the need for continuous monitoring or early detection is very crucial especially for facilities handling arsenic-containing wastes and sites, where arsenic is found at toxic levels in groundwater [1].

Due to its toxicity, the analysis of environmental sample by speciation procedure will identify and qualify the total quantity of arsenic present with their specific form. However, this method of analysis is expensive and requires sample collection methods to ensure the preservation of in situ conditions. Some other low-cost electrochemical methods such as stripping voltammetry or cyclic voltammetry [2]-[5]

Manuscript received September 19, 2015; revised November 20, 2015 This work was supported by the Ministry of Science, Technology and Innovation of Malaysia under research grant, Science Fund (06-03-04-SF0053).

The authors are with the Nano Semiconductor Technology, Mimos Berhad, Technology Park Malaysia, Kuala Lumpur 57000, Malaysia (e-mail nurulhaidahd@gmail.com, suraya.sulaiman@mimos.my, matek@mimos.my). have been used for detection of arsenic. Inert metal electrodes such as gold $(\mathrm{Au})$, carbon, and platinum $(\mathrm{Pt})$ have been used successfully for voltammetric detection of arsenic [6], [7]. Often these types of electrodes will be modified or incorporated with polymer, bio-element, or with another metal such as copper or cobalt to form an intermetallic compound with arsenic [8]. Each modification of electrode surface is expected to have higher electron kinetic transfer, higher sensitivity and stability over a wide range of solution composition, and a good reproducibility of the electrodes surfaces between each measurement.

Cobalt (Co) is known to be highly reactive and it has been employed in processes such as energy storage system [9], electrochromic thin films [10], and heterogeneous catalysis [11]. The electrocatalytic property of the cobalt very much depends on the deposition method. In order to create a good sensor response for in situ detection of arsenic, a possibility of cobalt electrodeposition on top of silicon-based platinum electrode was explored as to enhance the electron transfer signal.

The electrode sensitivity was characterized by cyclic voltammetry in a presence of As(III) solution. This paper only reported an initial study for electrochemical deposition of cobalt coating, especially in a different electrodeposition time for detection of As(III) solution. Later on, the nanostructure surface of cobalt coating can be modified with a functional group such as $-\mathrm{COOH},-\mathrm{OH}-\mathrm{NHs}$, thus they can be conjugated with active substances, which will be in favor of their application in biosensor fields [12], [13]. The electrode will be activated with bio-recognition element to make it more sensitive and selective to the target analyte.

\section{MATERIAL AND METHOD}

\section{A. Chemical and Reagents}

Solutions were prepared from analytical grade chemicals without further purification using distilled water. Boric acid solution $\left(\mathrm{H}_{3} \mathrm{BO}_{3}\right)$ and potassium hydroxide $(\mathrm{KOH})$ solution were prepared in $0.1 \mathrm{M}$ concentration. Cobalt(II) chloride $\left(\mathrm{CoCl}_{2}\right)$ and sodium arsenite $\left(\mathrm{NaAsO}_{2}\right)$ were of analytical grade.

\section{B. Modified Electrode and Apparatus}

In this electrodeposition study, silicon-based platinum electrode was used as a cathode or working electrode. The electrode area that was involved in deposition from aqueous solution within the surface of platinum is $1.8 \mathrm{~mm}^{2}$. $\mathrm{CoCl}_{2}$ dissolved in $0.1 \mathrm{M} \mathrm{H}_{3} \mathrm{BO}_{3}$ solution was reduced galvanostatically onto working electrode surface area using a 
$750 \mathrm{~W}$ single-output programmable basic system DC power supply from Agilent Technology (N5749A). Graphite was used as a counter electrode. All samples were prepared at $24^{\circ} \mathrm{C}$ with a current density of $0.50 \mathrm{~mA} / \mathrm{cm}^{2}$. Voltage given was $4.7 \mathrm{~V}$ and deposition time was varied (10s, 20s, and 30s) in order to have correlation between the quantity of electricity and the properties of the nanostructured film. The solution of reaction was stirred at $200 \mathrm{rpm}$ via magnetic stirrer to generate flow condition. All electrodes were cleaned in distilled water to remove traces of salts and air dried. The surface morphologies of the deposition electrodes were observed by field-emitting scanning electron microscopy (FESEM, Hitachi S-4800, Japan).

Electrodes were then characterized by using cyclic voltammetry method (Metrohm Autolab Potentiostats/Galvanostats (Model PGSTAT128N) to detect a redox signal in different concentration of As(III) solution. A three-conventional electrochemical cell was performed with Pt as counter electrode to conduct electricity from the signal source to the working electrode. All potentials are referred to a pseudo silver/silver chloride $(\mathrm{Ag} / \mathrm{AgCl})$ as reference electrode. Working electrode for cyclic voltammetry was made of cobalt oxide electrodeposited on platinum electrode (Pt-Co electrode). Voltammetry on modified electrodes was done in $0.1 \mathrm{M} \mathrm{KOH}$ supporting electrolyte containing no cobalt salt in a broad potential range. Electrochemical determination of arsenic in different concentration was done by a standard addition protocol and measured by NOVA 10.1 software.

\section{RESULT AND DISCUSSION}

\section{A. Characterization of Cobalt Deposition}

In electrodeposition process, the cobalt ions in a solution are reduced electrochemically to coat the surface of a conductive cathode known as platinum working electrode. Electrons follow to the cathode via an external electrical circuit. $\mathrm{H}_{3} \mathrm{BO}_{3}$ solution acts as a $\mathrm{pH}$ buffer to manipulate the surface $\mathrm{pH}$ on the cathode.

The result of FESEM study in Fig. 1 shows a correlation of cobalt grain diameter size in different time. As time deposition increased, larger drops in cobalt ions concentration were found on the electrode surface. The quantity and thickness of cobalt particles can be seen enhancing rapidly in 10-second (10s) difference of each deposition time. Deposited particles on the platinum surface are regularly separated and the size of cobalt nanostructured seems becoming larger by time. It can be seen that these small particles are almost uniformly distributed on the surface of electrode forming a thin layer. The particles sizes for 10s, 20s, and $30 \mathrm{~s}$ of deposition times are varied and can achieve maximum size to approximately $2.0 \mu \mathrm{m}, 4.9 \mu \mathrm{m}$, and $5.5 \mu \mathrm{m}$, respectively. The platinum electrode area was covered by a thick black material after 30 seconds of deposition time.

Fig. 2 shows FESEM cross-section result of a modified electrode for each deposition time studied. The average thickness of a packed grain size deposited in each 10s, 20s, and $30 \mathrm{~s}$ of time deposition are $14.7 \mu \mathrm{m}, 31.8 \mu \mathrm{m}$ and $61.3 \mu \mathrm{m}$, respectively. In 10 -second different time of deposition process, the grain diameter (as in Fig. 1) does not have a large difference in size but the thickness of the packed grain growth can achieve twice of its previous thickness. In Fig. $2 c$, the grain growth seems to be overlapping on each other and created a leaf shape of cobalt material. The dispersion of the packed grain in Fig. 2c is not even compared to a packed grain in Fig. $2 a$ and $2 b$. The thickness of a material deposited on the electrode surface can play a major role in the efficiency of electron transfer in redox reaction. A thicker type of growth would hinder the electron transfer between the analyte and the electrode surface in electrochemical analysis. Hence, only $10 \mathrm{~s}$ and 20 s of deposition times of modified electrodes were continued for arsenic detections.
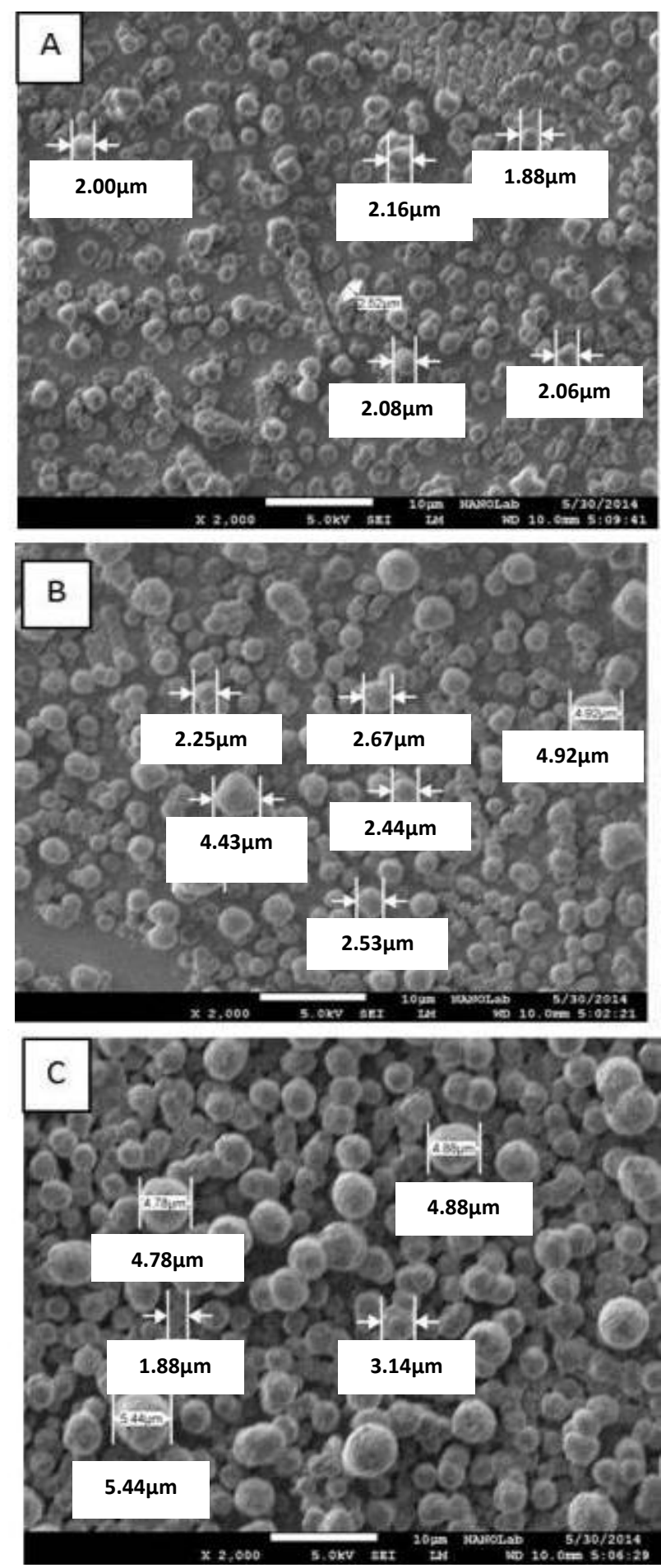

Fig. 1. FESEM micrograph of cobalt deposition on platinum electrode surface by electroplating in various times; (a) at $10 \mathrm{~s}$, (b) at 20s, and (c) at $30 \mathrm{~s}$. 

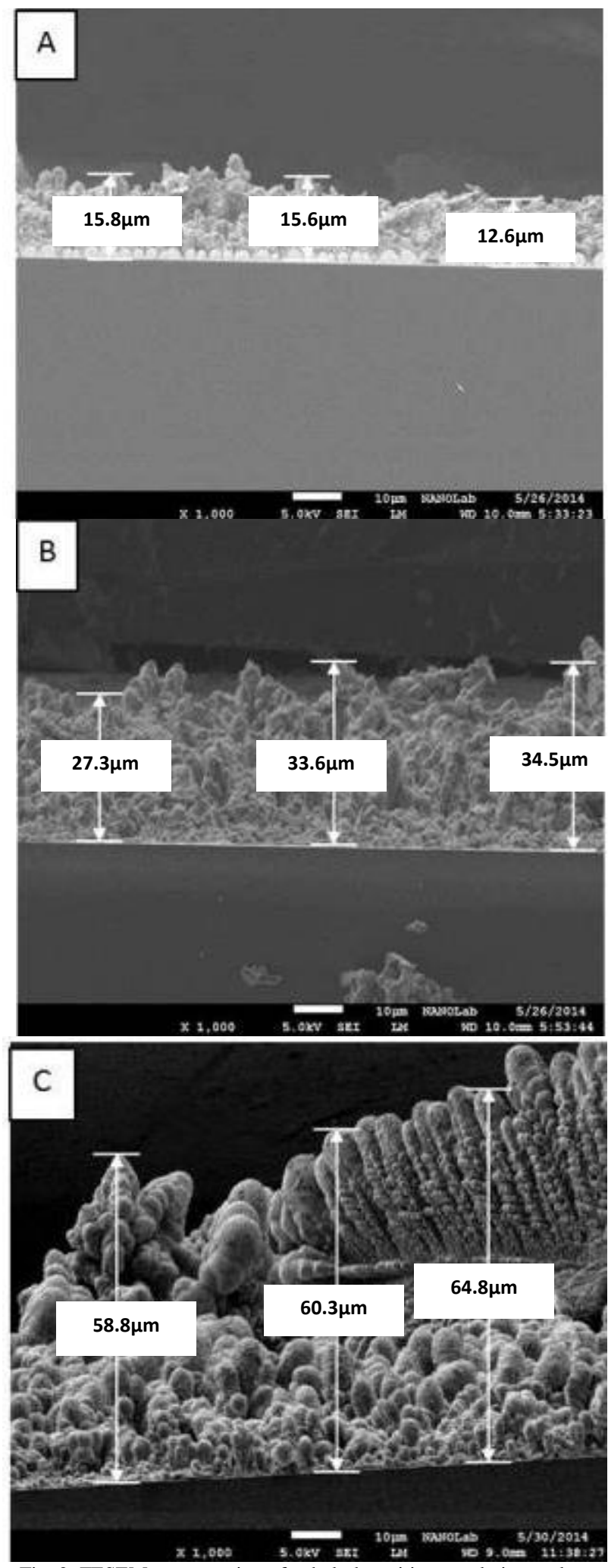

Fig. 2. FESEM cross-section of cobalt deposition on platinum electrode surface by electroplating in various times; (a) at $10 \mathrm{~s}$, (b) at 20s, and (c) at 30 s.

\section{B. Electrocatalytic Reaction of As(III) on Pt-Co Electrode}

The electrochemical experiments in the presence of different concentration of As(III) solution were carried out to evaluate the electrocatalytic activity of the modified Pt-Co electrode for arsenic redox reaction. The experiments were done on two types of cobalt deposition time; 10 s and 20s, based on FESEM surface morphology analysis.

In cyclic voltammetry method, the potential of deposited working electrode is measured against a pseudo reference electrode which maintains a constant potential, and the resulting applied potential produces an excitation signal of arsenic redox reaction. In forward scan of cyclic voltammetry of Fig. 3, a potential range was set between $-1.5 \mathrm{~V}$ to $+1.5 \mathrm{~V}$ for each scan at a scan rate of $0.1 \mathrm{~V} / \mathrm{s}$. Supporting electrolyte, $0.1 \mathrm{M} \mathrm{KOH}$, was used with addition of As(III) solution. Three different concentrations of As(III); 2, 8, and 16 ppm, were spiked orderly into a supporting electrolyte solution. Cyclic voltammetry was applied by measuring the current at the working electrode during the potential scan and voltammograms displayed a current versus potential graph.

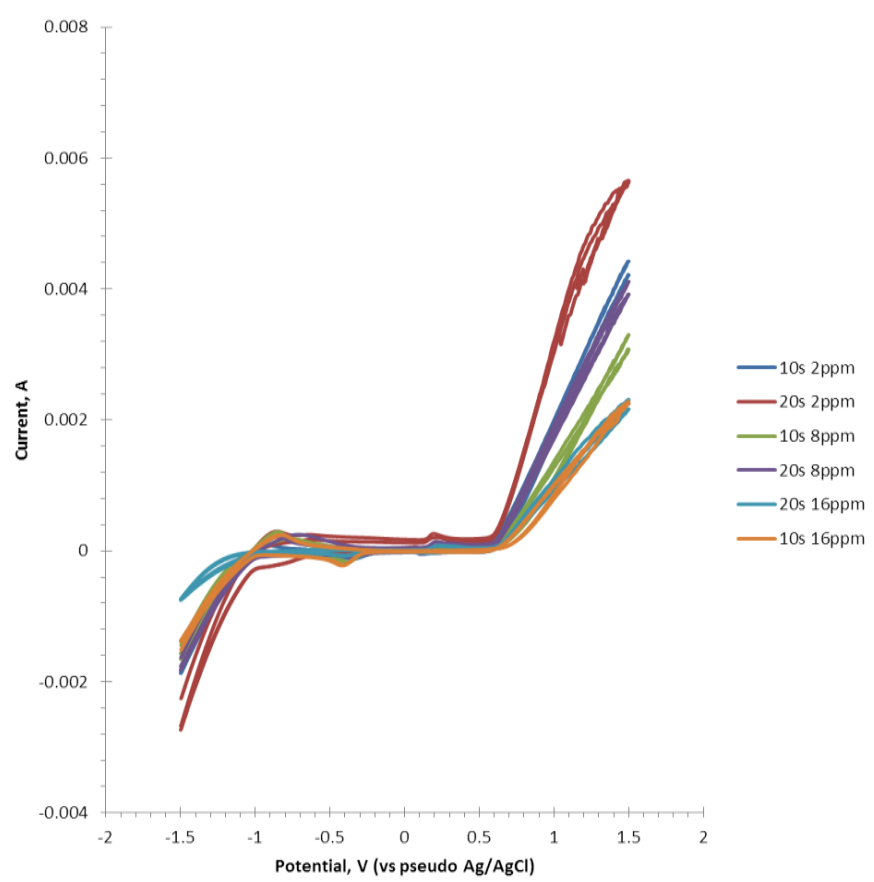

Fig. 3. Cyclic voltammogram of 10 s and 20s of Pt-Cobalt electrodeposition time with a varied concentration of As(III) solution ( $2 \mathrm{ppm}, 8 \mathrm{ppm}$, and 16 $\mathrm{ppm}$ ) in supporting electrolyte, $0.1 \mathrm{M} \mathrm{KOH}$, at a scan rate of $0.1 \mathrm{~V} / \mathrm{s}$. Potential ranges from $-1.5 \mathrm{~V}$ to $1.5 \mathrm{~V}$.

Fig. 3 shows cyclic voltammetry responses of a modified Pt-Co electrode with $10 \mathrm{~s}$ and 20 s of deposition time in different concentrations of As(III) solution. As a result of electrochemical Co-deposition of cobalt oxide on bare $\mathrm{Pt}$ electrode in arsenic solution, two main oxidation peaks are observed on the curve at $-0.9 \mathrm{~V}$ and $0.2 \mathrm{~V}$ in the presence of 2 to $16 \mathrm{ppm}$ of $\mathrm{As}(\mathrm{III})$. The first oxidation peak at $-0.9 \mathrm{~V}$ is attributed to the oxidation of $\mathrm{As}(0)$ to $\mathrm{As}(\mathrm{III})$ and the second one at $0.2 \mathrm{~V}$ corresponds to the oxidation of $\mathrm{As}(\mathrm{III})$ to $\mathrm{As}(\mathrm{V})$. Two reduction peaks appeared at $0.1 \mathrm{~V}$ and $-0.4 \mathrm{~V}$, suggesting a reduction of $\mathrm{As}(\mathrm{V})$ to $\mathrm{As}(\mathrm{III})$ and $\mathrm{As}(\mathrm{III})$ to $\mathrm{As}(0)$. In this study we focused on the second reduction peak (at $-0.4 \mathrm{~V}$ ) because the peak current is more sensitive in electrochemical detection of As(III).

The relativity of reduction current with $\mathrm{As}(\mathrm{III})$ concentration was studied by plotting a current versus As(III) concentration graph from Fig. 3 to find its correlation value on current peak height. Fig. 4 shows cyclic voltammograms and a graph of current versus As(III) concentration of $10 \mathrm{~s}$ and 20s of a modified Pt-Co electrodeposition time in supporting electrolyte, $0.1 \mathrm{M} \mathrm{KOH}$, at a scan rate of $0.1 \mathrm{~V} / \mathrm{s}$ and potential range from $-0.8 \mathrm{~V}$ to $+0.2 \mathrm{~V}$. The increased peak reduction current with increased concentration of As(III) solution 
confirms that cobalt particles have high catalytic ability for arsenic detection. They could act as a suitable mediator to shuttle electron between arsenic and working electrode. $R^{2}$ values from a linear regression equation obtained were 0.9976 and 0.9815 for a modified Pt-Co electrode deposition in $10 \mathrm{~s}$ and 20s, respectively.

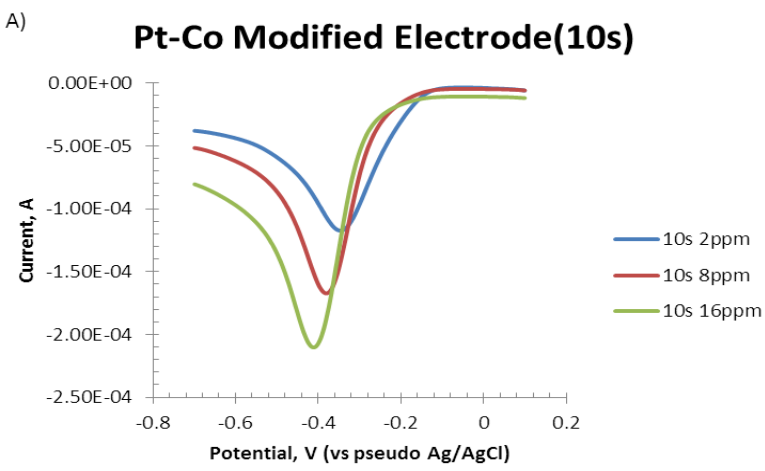

B)

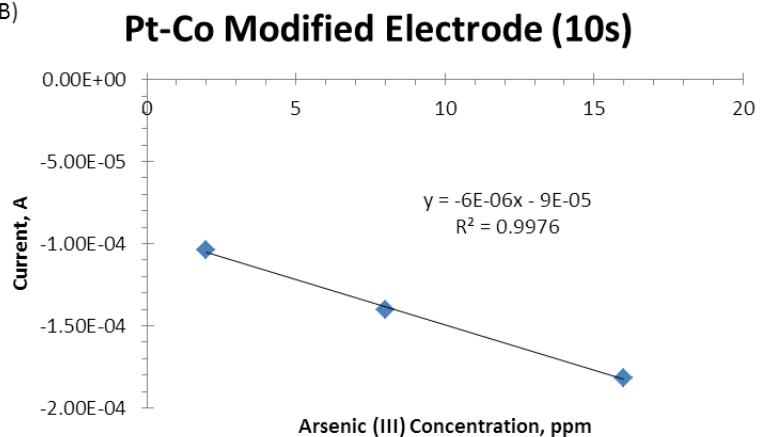

C)

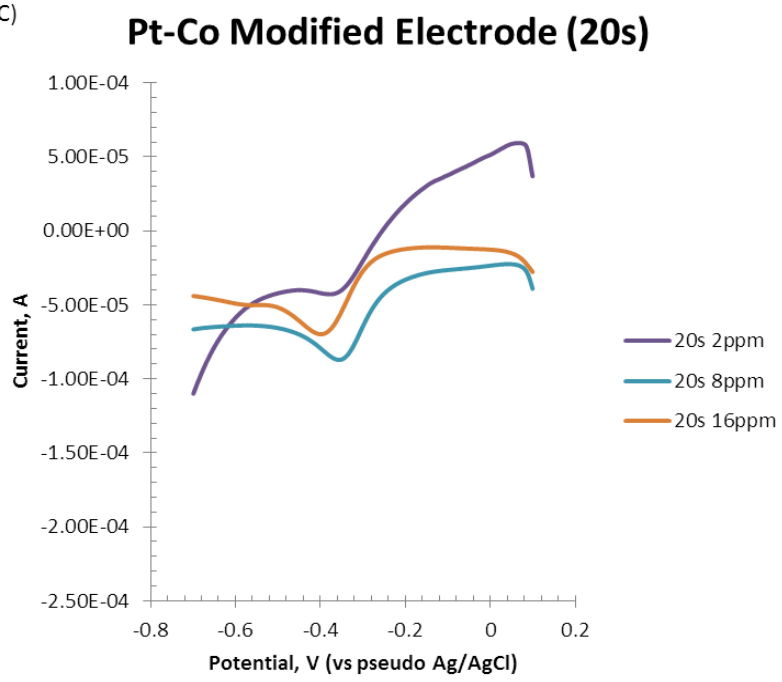

D)

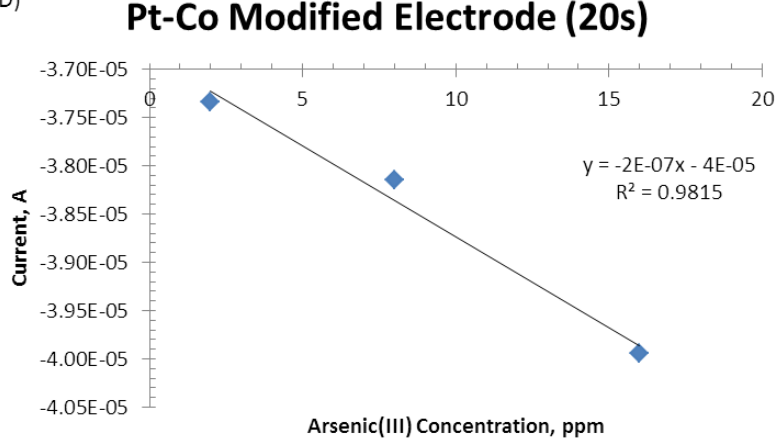

Fig. 4. Cyclic voltammogram and a graph of current versus As(III) concentration of $10 \mathrm{~s}$ and $20 \mathrm{~s}$ Pt-Co electrodeposition time in supporting electrolyte, $0.1 \mathrm{M} \mathrm{KOH}$, at a scan rate of $0.1 \mathrm{~V} / \mathrm{s}$. Potential ranges from -0.8
In testing conditions, the stable forms of $\mathrm{As}(\mathrm{III})$ and $\mathrm{As}(\mathrm{V})$ are $\mathrm{HAsO}_{2}$ and $\mathrm{H}_{3} \mathrm{AsO}_{4}$, respectively [14]. Based on the obtained voltammograms, the following catalytic scheme describes the possible associating reactions:

$$
\begin{gathered}
\mathrm{H}_{3} \mathrm{AsO}_{4}+2 \mathrm{H}^{+}+2 \mathrm{e}=\mathrm{HAsO}_{2}+2 \mathrm{H}_{2} \mathrm{O} \\
\mathrm{HAsO}_{2}+3 \mathrm{H}^{+}+3 \mathrm{e}=\mathrm{As}+2 \mathrm{H}_{2} \mathrm{O}
\end{gathered}
$$

The reduction peak current on a modified Pt-Co electrode with 10s deposition time is larger than 20s deposition time of modified Pt-Cobalt electrode. This fact demonstrates that the electrochemical deposition of cobalt ion on silicon-based $\mathrm{Pt}$ bare electrode in 10s can be a good approach for the detection of arsenic reduction. The current enhancement result suggested an attribution to the cooperative effect of a thinner cobalt deposition on bare Pt electrode where a thicker cobalt deposition is attributed to slow electron transfer and solution resistance in redox reaction.

The optimization of modified electrode for detection of arsenic and reduction peak behavior in different conditions will be continued later by our group. Their reduction peaks will be different in shape and peak potential mainly due to the electrode microstructural differences but electrodeposition of nanoparticle or material ions is still an attractive approach to fabricate sensor electrode as reported previously [15].

\section{CONCLUSION}

This study shows that the cobalt can be easily deposited on silicon-based platinum bare electrode with less deposition time and the modified electrode can be used for the electrochemical detection of As(III) via cyclic voltammetry. The reduction of $\mathrm{As}$ (III) based on the electrocatalytic cooperative effect of modified Pt-Co electrode showed a good response for the quantitative determination of As(III).

\section{ACKNOWLEDGMENT}

This work was supported by the Ministry of Science, Technology and Innovation of Malaysia under research grant, ScienceFund (06-03-04-SF0053).

\section{REFERENCES}

[1] K. G. Ajay and G. Mona, "Synthesis and surface engineering of iron oxide nanoparticles for biomedical applications," Biomaterials, vol. 26, p. 3995, 2005.

[2] H. Li and R. B. Smart, "Analysis of arsenic (V) by cathodic stripping voltammetry," Anal. Chim. Acta, vol. 306, p. 217, 1995.

[3] M. Kopanica and L. Novotny, "Determination of traces of Arsenic(III) by anodic stripping voltammetry in solutions, natural waters and biological material," Anal. Chim. Acta, vol. 368, p. 211, 1998.

[4] P. Salaun, B. Planer-Friedrich, and C. M. G. van den Berg, "Inorganic arsenic speciation in eater and seawater by anodic stripping voltammetry with a gold microelectrode," Anal. Chim. Acta, vol. 585, p. 312, 2007.

[5] J. Vandehecke, M. Waeles, R. Riso, and P.Corre, "A stripping chronopotentiometric (SCP) method with a gold film electrode for determining inorganic species in seawater," Anal. Bioanal. Chem., vol. 388, p. 929, 2007.

[6] E. Majid, S. Harapovic, Y. Liu, K. B. Male, and J. H. T. Luong, "Electrochemical determination of arsenite using a gold nanoparticle modified glassy carbon electrode and flow analysis, "Anal. Chem., vol. 78 , p. 762,2006 
[7] Y. Y. C. Sun, J. Mierzwa, and M. H. Yang, "New method of gold-film electrode preparation for anodic stripping voltammetric determination of Arsenic(III and V) in seawater, " Talanta, vol. 44, p. 1379, 1997.

[8] M. A. Ferreira and A. A. Barros, "Determination of As(III) and arsenic(V) in naturals waters by cathodic stripping voltammetry at a hanging mercury droelectrode, " Anal. Chim. Acta, vol. 459, p. 151, 2002.

[9] V. Srinivasan and J. W. Weidner, "Capacitance studies of cobalt oxide films-formed via electrochemical precipitation," J. Power Sources, vol. 108 , p. 15,2002

[10] L. D. kadam, S. H. Pawar, and P. S. Patil, "Studies on ionic interacalation properties of cobalt oxide thin films prepared by spray pyrolysis technique," Mater. Chem. Phys., vol. 68, p. 280, 2001.

[11] J. Tyczkowski, R. Kapica, and J. Lojewska, "Thin cobalt oxide film for catalysis deposited by plasma-enhanced metal -organic chemical vapor deposition," Thin Solid Films, vol. 515, p. 6590, 2007.

[12] D. T. Nguyen, D. J. Kim, and K. S. Kim, "Controlled synthesis and biomolecular probe application of gold nanoparticles," Micron, vol. 42, p. 207,2010

[13] X. K. Yan, Y. Sun, Y. Wu, B. Mayers, B. Gates, Y. Yin, F. Kim, and H. Yan, "One-dimensional nanostructures: Synthesis, characterization, and applications," Adv. Mater., vol. 15, p. 353, 2003.

[14] M. Pourbaix, Atlas of Electrochemical Equilibrium in Aqueous Solutions, Oxford, 1966, p. 516.

[15] A. Mukherjee et al., "Arsenic contamination in groundwater: A global perspective with emphasis on the Asian scenario," J. Health. Popul. Nutr., vol. 24, p. 142, 2006.

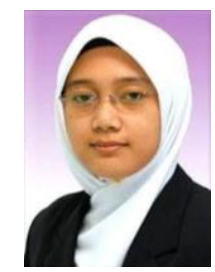

Nurulhaidah Daud graduated from Universiti Putra Malaysia for her $\mathrm{PhD}$ degree in analytical chemistry in 2012. She is currently working as Senior Researcher and part of Mems Lab and Technology Research Group in Nanosemiconductor Technology Department in MIMOS Berhad. She has been actively involved in various projects involving MIMOS's in house project and MOSTI SciendFund's project. Being exposed to a significant amount of project throughout her work thus far, Nurulhaidah embodies the value of teamwork and had gained some experienced in MEMS sensor. Her main research interests focus on biosensors and chemical sensors research. She has authored publications in local and international journals and proceedings.

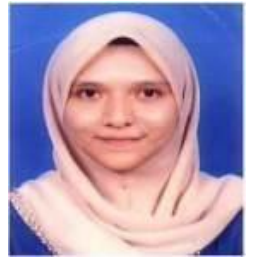

Nur Khairul Nabila Kamaruddin is currently completing her master of chemical science degree from Universiti Kebangsaan Malaysia. In June 2014, she joined MIMOS Berhad as Research Assistant for 1 year and obtained some experiences in sensor and Mems technology fabrication process. She specializes in polymer and chemical technology area and her passion is to have an opportunity to further her research in a $\mathrm{PhD}$ level.

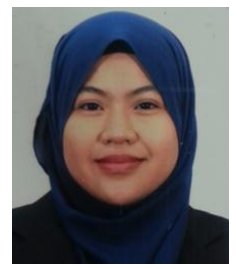

Suraya Sulaiman has obtained her bachelor degree of electrical, electronics and systems engineering and master of science degree from the Universiti Kebangsaan Malaysia in 2003 and 2008 respectively. She did her research in Nanoelectronics Department, in MIMOS Berhad from year 2003 till current. She has published many papers in the local and international conferences. She has jointly filed more than 10 patents. Her scientific interests include MEMS sensor, nano sensor, testing and sensor characterization for agriculture, environmental and medical applications

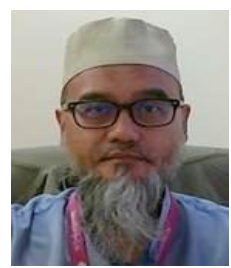

Mohd Ismahadi Syono obtained his bachelor and master degree in physics from Indiana University of Bloomington and Boston College in 1986 and 1988 respectively and his $\mathrm{PhD}$ degree in microelectronics from Universiti Kebangsaan Malaysia in 2007. He started his career on development of voltage contrast technique for failure analysis followed by development of semiconductor devices fabrication \& characterization. He is involved in technology transfer and qualification of $1 \mathrm{um}$ CMOS process and subsequently attached to various other depts in the division. In 2005, he led the development of MEMS roadmap for Malaysia and appointed head of research division on Nanotechnology \& Photonics. His current research interest is in smart sensor arrays for medical \& healthcare applications. 